\title{
Direct microscopy versus sputum cytology analysis and bleach sedimentation for diagnosis of tuberculosis: a prospective diagnostic study
}

\author{
Pamela Hepple ${ }^{1 *}$, Pascal Nguele ${ }^{2}$, Jane Greig ${ }^{1}$, Maryline Bonnet ${ }^{3}$, Vinciane Sizaire ${ }^{1}$
}

\begin{abstract}
Background: Diagnostic options for pulmonary tuberculosis in resource-poor settings are commonly limited to smear microscopy. We investigated whether bleach concentration by sedimentation and sputum cytology analysis (SCA) increased the positivity rate of smear microscopy for smear-positive tuberculosis.

Methods: We did a prospective diagnostic study in a Médecins Sans Frontières-supported hospital in Mindouli, Republic of Congo. Three sputum samples were obtained from 280 consecutive pulmonary tuberculosis suspects, and were processed according to WHO guidelines for direct smear microscopy. The remainder of each sputum sample was homogenised with $2.6 \%$ bleach, sedimented overnight, smeared, and examined blinded to the direct smear result for acid-fast bacilli (AFB). All direct smears were assessed for quality by SCA. If a patient produced fewer than three good-quality sputum samples, further samples were requested. Sediment smear examination was performed independently of SCA result on the corresponding direct smear. Positivity rates were compared using McNemar's test.

Results: Excluding SCA, 43.2\% of all patients were diagnosed as positive on direct microscopy of up to three samples. $47.9 \%$ were diagnosed on sediment microscopy, with $48.2 \%$ being diagnosed on direct microscopy, sediment microscopy, or both. The positivity rate increased from $43.2 \%$ to $47.9 \%$ with a case definition of one positive smear ( $\geq 1 \mathrm{AFB} / 100$ high power fields) of three, and from $42.1 \%$ to $43.9 \%$ with two positive smears. SCA resulted in $87.9 \%$ of patients producing at least two good-quality sputum samples, with $75.7 \%$ producing three or more. Using a case definition of one positive smear, the incremental yield of bleach sedimentation was 14/121, or $11.6 \%(95 \% \mathrm{Cl} 6.5-18.6, \mathrm{p}=0.001)$ and in combination with SCA was $15 / 121$, or $12.4 \%(95 \% \mathrm{Cl} 7.1-19.6, \mathrm{p}=0.002)$. Incremental yields with two positive smears were $5 / 118$, or $4.2 \%(95 \% \mathrm{Cl} 1.4-9.6, \mathrm{p}=0.062)$ and $7 / 118$, or $5.9 \%$ (95\% Cl 2.4-11.8, $\mathrm{p}=0.016)$, respectively.

Conclusions: The combination of bleach sedimentation and SCA resulted in significantly increased microscopy positivity rates with a case definition of either one or two positive smears. Implementation of bleach sedimentation led to a significant increase in the diagnosis of smear-positive patients. Implementation of SCA did not result in significantly increased diagnosis of tuberculosis, but did result in improved sample quality. Requesting extra sputum samples based on SCA results, combined with bleach sedimentation, could significantly increase the detection of smear-positive patients if routinely implemented in resource-limited settings where gold standard techniques are not available. We recommend that a pilot phase is undertaken before routine implementation to determine the impact in a particular context.
\end{abstract}

\footnotetext{
* Correspondence: pamela.hepple@london.msf.org

'Manson Unit, Médecins Sans Frontières UK, 67-74 Saffron Hill, London EC1N

$8 \mathrm{QX}, \mathrm{UK}$

Full list of author information is available at the end of the article
}

\section{Biomed Central}

C 2010 Hepple et al; licensee BioMed Central Ltd. This is an Open Access article distributed under the terms of the Creative Commons Attribution License (http://creativecommons.org/licenses/by/2.0), which permits unrestricted use, distribution, and reproduction in any medium, provided the original work is properly cited. 


\section{Background}

Tuberculosis (TB) is a major public health problem, with an estimated 2 billion people infected with tubercule bacilli worldwide. Estimated global prevalence of the disease is 139 per 100,000 population [1]. In recent years, the prevalence of TB has increased steeply, driven in large part by the HIV epidemic. TB in patients co-infected with HIV can be difficult to diagnose, especially by sputumsmear microscopy-often the only diagnostic tool available in resource-limited settings. The medical humanitarian aid agency Médecins Sans Frontières (MSF) works in settings where the health infrastructure needed to treat patients with TB, many of whom are co-infected with HIV, is either absent or extremely limited.

The gold standard for diagnosing pulmonary tuberculosis is culture of sputum on Löwenstein-Jensen medium. However, due to lack of access to culture facilities and the long turn-around times involved with sputum culture, most programmes use direct Ziehl-Neelsen microscopy for detection of acid-fast bacilli (AFB) in sputum smears. This technique, when performed optimally, has reported sensitivities ranging from $61.8 \%$ to $70 \%$ compared with the gold-standard [2-4]. Sensitivity is reduced if samples are of poor quality, which is often the case in children and in people who are HIV-positive, since sputum expectoration is more difficult $[3,5]$.

Sputum samples are also often inadequate in patients who are immune-compromised or who have not been given correct instructions on sputum expectoration [6]. Patients frequently provide pure saliva or very small amounts of sputum in saliva instead of an appropriate, purulent, sputum sample [7]. A negative result issued on examination of such a sample is misleading, since it implies that a correct sputum specimen has been examined. Sputum cytology analysis (SCA), in which smears are examined and graded according to an algorithm, has been proposed as a means to ensure sufficient sample quality. Further samples can be then requested if quality is inadequate [8].

Sputum concentration by homogenisation and microscopic examination of the sediment can increase the detection rate of AFB. Several concentration techniques using sedimentation or centrifugation have been reported [9-11]. Bleach homogenisation followed by overnight sedimentation can significantly increase detection of AFB compared with direct microscopy [12-14]. A systematic review of this technique found an increase in sensitivity of $33 \%$ in one of four studies where culture was used as a gold standard, and a mean incremental yield of $6 \%$ in four studies in which culture was not used [9]. Centrifugation resulted in higher yields than sedimentation. Cattamanchi and colleagues [15] found that bleach concentration resulted in small increases in sensitivity and small decreases in specificity. However, centrifugation requires electricity as well as equipment that might not be present in peripheral laboratories in resource-limited settings. Homogenisation can be achieved with various agents, such as sodium hydroxide $(\mathrm{NaOH})$ and $\mathrm{N}$-acetyl- $\mathrm{L}$ cysteine (NALC) [11]. Household bleach-sodium hypochlorite $(\mathrm{NaOCl})-\mathrm{can}$ also be used, and it has the advantage of being easily available worldwide.

Due to the variability of results to date, it has been recommended that further research is done before the adoption of bleach concentration as a standard diagnostic method [10].

We did a prospective field assessment in routine conditions in a peripheral laboratory in an MSF-supported hospital in Mindouli, Republic of Congo. We aimed to compare the proportion of smear-positive patients detected with and without bleach concentration of sputum by overnight sedimentation with validation of all samples by SCA. Our hypothesis was that the combination of SCA and bleach sedimentation would significantly improve the detection rate for smear microscopy of pulmonary TB.

\section{Methods}

\section{Setting and patients}

The study was conducted between October 2006 and March 2008 in Mindouli, Pool Region, Republic of Congo. MSF was supporting the district Ministry of Health hospital, with services ranging from outpatient care, maternal care, and treatment of infectious diseases such as tuberculosis and HIV/AIDS, to psychosocial counselling and emergency surgery. Fighting between rebels and government forces meant that health care services had been neglected in the region, and food insecurity had resulted in high rates of malnutrition. After a ceasefire, internally displaced people returned to the region, leading MSF to start interventions in Ministry of Health hospitals in Kinkala, Kindamba, and Mindouli. Adult HIV prevalence is less than $4 \%$, with a TB prevalence of 449 per 100,000 population [16,17]. Patients were included according to the following criteria: 15 years of age or older; suspected pulmonary TB with cough of at least 3 weeks duration; and non-ingestion of anti-TB drugs in the previous month. Every patient suspected of having pulmonary TB was assessed by a TB nurse and invited to participate in the study. An informed consent form was signed by those who agreed to participate. All patients invited to participate agreed to do so. Patients were accommodated in the hospital until a final result was available, according to standard procedures in the project setting.

\section{Ethics approval}

Ethics approval for the study was obtained from the MSF Ethics Review Board. The study was also approved 
by the Ministry of Health Provincial Directorate of the National TB Programme of the Republic of Congo.

\section{Specimen collection and processing}

Patients were given instructions on sputum production. Collection of the first sample was supervised by a TB nurse. Samples lacking any purulent material were rejected and the patient asked to try again. Patients were given a sputum container for expectoration at home the following morning. When the second sample was brought to the laboratory, it was examined macroscopically. If no purulent material was present, a laboratory technician supervised collection of a replacement sample. A third container was provided for expectoration the following morning. After processing, if there were fewer than three good sputum samples the patient was asked to provide further spot samples.

Results were passed on to the clinician once all requested samples had been processed for direct microscopy and independently of results of sediment microscopy. For the case definition of one positive sample, any positive sample in the sample batch was considered eligible. For the case definition of two positive samples, any two positive samples in the sample batch were considered eligible. Direct microscopy smears were processed according to the standard hot Ziehl-Neelsen staining procedure and examined for SCA and presence of AFB.

After direct smears had been made, the remainder of each sample was processed for bleach sedimentation according to the procedure outlined by Bonnet and colleagues [11], with the exception that samples were manually shaken for 30 seconds instead of being vortexed. In brief, a direct smear was made from each sputum sample. An equal amount of household bleach was then added to the sputum sample in a screw-cap tube, shaken for 30 seconds, and allowed to sediment overnight. The following morning, the supernatant was decanted and a smear was made of the sediment. Samples were then processed according to the standard hot Ziehl-Neelsen staining procedure, and the smears were examined for presence of AFB.

Both direct and sediment smears were examined in a blinded manner by two laboratory technicians, and graded according to the Pasteur scale, which was then converted to the WHO-IUATLD scale. In case of discordant results, both readers repeated the slide reading until a consensus was reached. Monthly blinded quality control (QC) was done by the laboratory supervisor using five positive and five negative randomly selected smears from both direct and sediment subsets. Direct slides were also assessed for sputum cytology analysis agreement. External blinded QC was done halfway through and at the end of the study on $10 \%$ of positive and $10 \%$ of negative samples in both subsets. QC for SCA was also done on $10 \%$ of direct smears.

\section{$\mathrm{NaOCl}$ solution}

"Lacroix" bleach was purchased from a supermarket in Brazzaville, Republic of Congo. Enough was purchased for the study duration. The stated chlorine concentration was $2.6 \%$. To prevent reduction of chlorine activity from repeated exposure to air [18], each $2.5 \mathrm{~L}$ bottle was decanted on opening into $25 \mathrm{~mL}$ brown glass bottles for daily use. Each new $2.5 \mathrm{~L}$ bottle was tested for residual chlorine availability using the Palintest ( $R$ ) diethyl-p-phenylene diamine (DPD) pool testing system, with the bleach in a 1:10,000 dilution. The bleach was always in the range of $1-1.5 \mathrm{mg} / \mathrm{L}$ at this dilution. A small bottle was randomly selected each week for testing with DPD, at the same dilution, and was always between 1-1.5 $\mathrm{mg} / \mathrm{L}$, indicating no reduction of activity. A Ziehl-Neelsen smear was made from each new bottle to ensure no contamination with mycobacteria.

\section{Sputum cytology analysis}

Smears were examined with $\times 10$ objective $(\times 100$ magnification) and categorised according to the SCA algorithm (Table 1).

\section{Sample size}

For a power of $80 \%$ at 0.05 precision, and an expected increase in positivity from $27 \%$ to $32 \%$ with SCA and bleach sedimentation, the sample size required was 543 patients (McNemar test).

\section{Data analysis}

Data were double-entered in Excel 2000, cross-checked, and analysed using Excel 2000 and STATA v10 (College Station, Texas, USA). The exact McNemar's test was used to compare matched data for direct versus sediment results, using a case definition of either one or two positive results. A positive result was defined as $\geq 1$ AFB per 100 high-power field. Analysis was done on the outcome based on obtaining one or two positive samples to reflect a change in the 2007 WHO recommendations that occurred halfway through the study [19]. We analysed the smear-positive detection rate of several combinations of direct and bleach sedimentation microscopy to identify the optimal algorithm for detection of smear positives, with a case definition of one positive smear and using two samples. An alpha of 0.05 was considered significant, and exact binomial 95\% CIs were calculated.

\section{Results}

Patient enrolment progressed more slowly than expected, with only 280 patients enrolled after an 
Table 1 Sputum cytology analysis definitions [8]

\begin{tabular}{ll}
\hline & Definition \\
\hline Saliva & $\begin{array}{l}\text { Presence of squamous epithelial cells and/or dark patches; absence of particulate material, absence of } \\
\text { significant number of polymorphoneutrophils/macrophages }\end{array}$ \\
Mucus & $\begin{array}{l}\text { Acellular, defined edges } \\
\text { Degraded sputum }\end{array}$ \\
& $\begin{array}{l}\text { Presence of dark patches of material on } \times 10 \text { objective which, using } \times 100 \text { objective, are totally covered with } \\
\text { blue-staining non-mycobacterial bacteria; presence of polymorphoneutrophils/macrophages and/or fibrils }\end{array}$ \\
$\begin{array}{l}\text { Insufficient sputum (good quality, } \\
\text { insufficient quantity) }\end{array}$ & $\begin{array}{l}\text { Presence of patches of polymorphoneutrophils/macrophages and/or fibrils, but filling fewer than ten fields } \\
\text { "Good/real" sputum (good quality, } \\
\text { good quantity) }\end{array}$ \\
\hline
\end{tabular}

extension of the study from 1 year to 18 months. The total number of samples obtained was 890 . Of the 280 patients enrolled, $41 \%$ (115) were male. The mean age was 35 years (SD 12.1). Nine patients $(3.2 \%)$ were lost to follow up, and three patients died.

Most patients $(223 / 280)$ provided three samples. $49 /$ 280 provided four or more and $8 / 280$ provided fewer than three.

Table 2 shows the detection rates of the different techniques and the incremental yield of bleach sedimentation and SCA over direct microscopy alone. Using bleach sedimentation and a case definition of one positive smear, 14 extra patients were detected, which was $10.4 \%$ (95\% CI 5.8-16.8) of all positive patients detected (without SCA); this was an incremental yield of 14/121, or $11.6 \%$ (95\% CI 6.5-18.6) (Table 2). One patient was positive on direct microscopy and negative on sediment microscopy, resulting in a total of 121 patients diagnosed by direct microscopy, of which 120 were positive on sediment microscopy. The incremental increase of sediment microscopy only was $10.7 \%$ (95\% CI $5.8-17.7$ ), meaning there would have been fewer patients (1) detected if direct microscopy had not been used. The combined use of bleach sedimentation and SCA gave an incremental yield of $15 / 121$, or $12.4 \%$ (95\% CI 7.1-19.6; Table 2).

When a case definition of two positive smears was used, bleach sedimentation gave an incremental yield of $5 / 118$, or $4.2 \%$ (95\% CI 1.4-9.6; Table 2). Only five extra patients were detected-4.1\% (95\% CI 1.3-9.2) of all patients detected as positive with this case definition. After SCA, two extra patients were detected on sedimentation but none on direct microscopy. The incremental yield of both techniques was $7 / 118$, or $5.9 \%$ (95\% CI 2.4-11.8; Table 2).

Results were analysed to determine the optimal algorithm for detection of smear positives, with a case definition of one positive smear and using two samples (Table 3). Sedimentation of both sample 1 and 2 would have resulted in the highest positivity rate (46.8\%). A direct smear from the first sample, followed by sedimentation of the second, was almost as effective $(45.4 \%)$. The incremental case detection of $\mathrm{B} 1+\mathrm{B} 2$ (sedimentation of first and second sample) compared with $\mathrm{D} 1+\mathrm{D} 2$ (direct smears for both) would have been $11 / 120(9.2 \%)$.

$83 \%(737 / 890)$ of samples, including extra samples requested after SCA, were good-quality sputum. 15\% (137/890) were insufficient or degraded sputum. 2\% (16/ 890 ) of samples were saliva or mucus. One patient failed to produce any sputum samples, but was retained in the study (three saliva samples, negative on direct, all positive on sediment).

All poor-quality samples (degraded, mucus, and saliva) were negative on direct microscopy. Using bleach sedimentation on 47 poor-quality samples resulted in seven positive results (18.4\%: four [10.5\%] for degraded, three [7.9\%] for saliva), which improved the overall detection

Table 2 Detection rate of different techniques and comparison of incremental yield over direct microscopy alone

\begin{tabular}{|c|c|c|c|c|}
\hline & \multicolumn{2}{|c|}{ Case definition of one smear } & \multicolumn{2}{|c|}{ Case definition of two smears } \\
\hline & $\begin{array}{l}\mathrm{N} \text { detected }(\% \text { of all } \\
\text { patients) }\end{array}$ & $\begin{array}{l}\text { Incremental yield over D alone: N (\%; } \\
95 \% \mathrm{Cl} \text { ) }\end{array}$ & $\begin{array}{l}\mathrm{N} \text { detected (\% of all } \\
\text { patients) }\end{array}$ & $\begin{array}{l}\text { Incremental yield over D alone: N (\%; } \\
95 \% \mathrm{Cl})\end{array}$ \\
\hline D & $121(43.2 \%)^{*}$ & - & $118(42.1 \%)$ & - \\
\hline $\begin{array}{l}\mathrm{D} \\
+\mathrm{SCA}\end{array}$ & $122(43.6 \%)$ & $1(0.8 \% ; 0-4.5)$ & $118(42.1 \%)$ & 0 \\
\hline B & $134(47.9 \%) \dagger$ & $14(11.6 \% ; 6.5-18.6)$ & $123(43.9 \%)$ & $5(4.2 \% ; 1.4-9.6)$ \\
\hline $\begin{array}{l}\mathrm{B} \\
+\mathrm{SCA}\end{array}$ & $135(48.6 \%) \neq$ & $15(12.4 \% ; 7.1-19.6)$ & $125(44.6 \%)$ & 7 (5.9\%; 2.4-11.8) \\
\hline
\end{tabular}

$\mathrm{D}=$ direct microscopy. SCA = sputum cytology analysis. $\mathrm{B}=$ bleach sedimentation. ${ }^{*}$ One patient was positive on direct microscopy and negative on sediment microscopy, resulting in a total of 121 patients diagnosed by direct microscopy, of which 120 were positive on sediment microscopy. tOne patient positive on direct but negative on sediment in three samples (without SCA). $\neq$ One patient positive on sediment but not on direct microscopy after SCA. 
Table 3 Smear-positive detection rates of direct and bleach smear microscopy (excluding SCA)*

\begin{tabular}{lcccc}
\hline Approach & \multicolumn{3}{c}{ Smear-positive detection rate } & Compared with D1+D2 \\
& $\mathbf{n}$ & $\mathbf{\%}$ & $\mathbf{9 5 \%} \mathbf{C l}$ & $\mathbf{p}$ \\
\hline $\mathrm{D} 1+\mathrm{D} 2$ & 120 & $\mathbf{4 2 . 9}$ & $37.0-48.9$ & - \\
$\mathrm{B} 1$ & 125 & $\mathbf{4 4 . 6}$ & $38.7-50.7$ & 0.23 \\
$\mathrm{~B} 1+\mathrm{B} 2$ & 131 & $\mathbf{4 6 . 8}$ & $40.8-52.8$ & $<0.01$ \\
$\mathrm{D} 1+\mathrm{B} 1$ & 128 & $\mathbf{4 5 . 7}$ & $39.8-51.7$ & 0.01 \\
$\mathrm{~B} 1+\mathrm{D} 2$ & 127 & $\mathbf{4 5 . 4}$ & $39.4-51.4$ & 0.04 \\
$\mathrm{D} 1+\mathrm{B} 2$ & 127 & $\mathbf{4 5 . 4}$ & $39.4-51.4$ & 0.02 \\
$\mathrm{D} 1+\mathrm{B} 1+\mathrm{D} 2$ & 128 & $\mathbf{4 5 . 7}$ & $39.8-51.7$ & 0.01 \\
$\mathrm{D} 1+\mathrm{D} 2+\mathrm{B} 2$ & 127 & $\mathbf{4 5 . 4}$ & $39.4-51.4$ & 0.02 \\
$\mathrm{D} 1+\mathrm{B} 1+\mathrm{B} 2$ & 132 & $\mathbf{4 7 . 1}$ & $41.2-53.2$ & $<0.01$ \\
$\mathrm{~B} 1+\mathrm{D} 2+\mathrm{B} 2$ & 127 & $\mathbf{4 5 . 4}$ & $39.4-51.4$ & 0.02 \\
\hline $\mathrm{D}$ & &
\end{tabular}

$\mathrm{D}=$ direct. $\mathrm{B}=$ bleach sedimentation. ${ }^{*}$ Based on the $2007 \mathrm{WHO}$ smearpositive case definition (one smear positive with $\geq 1 \mathrm{AFB} / 100$ high-power field).

rate $(\mathrm{p}=0.0215)$. The proportion of good-quality samples giving a positive result was significantly more using sedimentation (360 of 890) than direct microscopy (347 of $890 ; \mathrm{p}=0.0044$ ).

Table 4 shows the number of patients able to produce good-quality sputum samples before and after SCA. Nine patients were able to provide at least two good samples after SCA (3.8\%; 95\% CI 2.8-7.1) and 17 patients provided three or more $(10.8 \%$; $95 \%$ CI 6.8 16.0). Of 68 patients unable to produce three or more good-quality samples, $30(44.1 \%)$ could not produce further samples, despite repeated efforts; three (4.4\%) died before further samples could be requested; nine (13.2\%) were lost to follow-up; and data for 26 patients (38.2\%) are missing.

\section{Quality control}

A total of 1780 slides were read. Fewer than ten interreader discrepancies were noted and all were resolved upon re-reading. External QC results showed excellent agreement for direct and bleach sedimentation techniques (both 99\%), with very good agreement for SCA (90\%).

\section{Discussion}

Our results show that bleach sedimentation and SCA increased the detection of smear-positive TB patients by $12.4 \%$, which is in line with the findings of other studies reporting incremental yields ranging from $7 \%$ to $253 \%$ [20]. A meta-analysis found that, in studies comparing overnight sedimentation with culture, the mean increase in sensitivity was $23 \%$, whereas in those without culture, the mean incremental yield was 5\% [9].

The main implication of our study is that implementation of bleach sedimentation would significantly increase the detection of smear-positive patients if routinely implemented in resource-limited settings where gold standard techniques are not available.

SCA is a low-workload intervention which takes roughly $15-30$ seconds to perform, and so can easily be integrated into routine examination. Our QC results show that the technique is highly reproducible. However, bleach sedimentation increased workload and delayed results by 1 day compared with direct microscopy.

The combination which yielded the highest positivity (45.4\%), using a maximum of two samples and incurring minimal extra workload and delay, was a direct smear for the first sample and bleach sedimentation for the second. This approach allows rapid identification of patients positive on the first direct smear without waiting for the results of sedimentation. The use of two samples (the supervised sample and the one obtained the next morning) increased sensitivity by allowing for one sample to be of poor quality, and by concentrating the sample which is likely to be of the best quality (the morning sample). This procedure is based on the 2007 WHO recommendations [19,21].

One patient detected using direct smear microscopy was negative on sediment smear. This result could have been caused by chance, with the only AFB-containing portion of the sputum being used to make the direct smear.

The analyses of residual chlorine activity showed that there was no reduction of chlorine activity after the bleach was decanted into small bottles. Because homogenisation activity is also visible microscopically, (i.e. lack of homogenisation results in intact white blood cells after sedimentation), it should not be necessary to measure residual chlorine activity routinely.

Sediment smears were fragile and easily washed off the slides during staining. Attempts to prevent this with the addition of bovine serum albumin made smears difficult to decolourise, and the practice was discontinued. AFB in sediment smears were also found to fade; we

Table 4 Number of patients able to produce good-quality sputum samples before and after SCA

\begin{tabular}{llll}
\hline & $\begin{array}{l}\text { Able to produce three samples ( } \mathbf{n} \\
[\%])\end{array}$ & Able to produce two or more samples (n [\%]) & $\begin{array}{l}\text { Unable to produce any samples (n } \\
[\%])\end{array}$ \\
\hline $\begin{array}{l}\text { Before } \\
\text { SCA }\end{array}$ & $195(69.6 \%)$ & $237(84.6 \%)$ & $12(4.3 \%)$ \\
After SCA & $212(75.7 \%)$ & $246(87.9 \%)$ & $9(3.2 \%)$ \\
\hline
\end{tabular}

SCA = sputum cytology analysis. 
therefore recommend that $\mathrm{QC}$ is performed within 6 months of staining.

Our results showed that poor-quality samples are more likely to be negative on direct and sediment microscopy than good-quality samples, and that both types are significantly more likely to be positive after bleach sedimentation than on direct microscopy. 10.5\% of degraded samples were negative in direct microscopy and positive on sedimentation. Although the sample size was small (4 of 38), this result suggests a need for further investigation. With the three saliva samples that became positive after sedimentation, a small amount of sputum was probably present.

A recent study has stated that macroscopic evaluation of sputum for quality is as effective as microscopic techniques such as SCA [22]. The study highlighted that rejection of samples based on cytological criteria would have resulted in some positive samples being missed. However, SCA focuses on the use of cytological classification to request extra samples when negative samples are of poor quality, and all smears are examined for AFB irrespective of quality. Although the actual number of increased smear-positive patients detected with SCA was low in our study, it can be inferred that the negative results obtained with good-quality samples are more meaningful than those from poor-quality samples. The clinician can be reassured that an appropriate patient sample was examined, due to cytological confirmation of the presence of sputum. The QC results for SCA showed agreement of $90 \%$, which was lower than the 99\% seen for AFB sensitivity on both direct and sediment smears. This result was expected, since SCA is less objective than observing the presence or absence of AFB. However, 90\% agreement indicates that the results are highly reproducible.

The patient positivity rate with direct microscopy without SCA was $43.2 \%$. This rate is considerably higher than the recommended positivity rate of $5-20 \%$ [23] and implies that case detection was not optimal-i.e. that the suspects referred for testing were likely to be more strongly positive than in a standard patient population. This was probably because of a selection bias in the patients referred for TB diagnosis. The hospital functioned as an unofficial referral hospital for people living with HIV/AIDS, and very sick patients often selfreferred from all over the country. These patients could have had advanced TB infections. A larger incremental yield for bleach sedimentation and SCA might have been observed if the positivity rate was lower, and patients had been in less advanced stages of infection.

The main limitation of our results is that, due to a lack of TB culture facilities, we were unable to incorporate the gold standard of TB diagnosis: culture on Lowenstein-Jensen medium. We are therefore unable to describe the techniques with regard to increased sensitivity and potential false smear positive results, but only with regard to increased detection rate of AFB or incremental yield. Lack of culture confirmation could have resulted in false detection of positive results, which might have contributed to the incremental yield following sedimentation. The sample size was not reached despite extending the study to 18 months; unfortunately, logistical limitations prevented extending the study further. Bleach sedimentation with a case definition of two positive smears might have significantly increased yield if the required sample size had been reached. However, most results reached significance despite the lower-than-desired number of participants.

Mindouli hospital functioned as a comprehensive HIV diagnostic and treatment centre, and a large proportion of those screened for TB infection were HIV positive. Although it would have been interesting to have stratified the results based on HIV status, this was not done due to concerns about patient confidentiality if TB results were linked with HIV results at the laboratory. We are therefore unable to provide an estimate for the proportion of HIV TB coinfected patients.

Our loss-to-follow-up rate was low (3.2\%), possibly because of emphasis on patient education during enrolment and provision of accommodation during the 3-day sample collection procedure, necessary since many patients came from outside the Mindouli area. Under routine conditions, without the provision of accommodation, a higher proportion of patients might be lost to follow up. The overnight delay in results following sedimentation could also lead to a higher patient loss to follow up. The loss to follow up rate after routine implementation should be monitored.

Due to the disadvantages associated with the bleach concentration technique (i.e. fragility of sediment smears, delayed results, and increased workload), routine implementation of the technique should only be considered after the feasibility of introduction in a particular context has been assessed, preferably following a pilot implementation phase.

\section{Conclusion}

Implementation of bleach sedimentation will lead to a significant increase in the diagnosis of smear-positive TB patients. Implementation of SCA did not result in significantly increased diagnosis of TB. Efforts should be made to obtain good-quality samples, since bleach sedimentation of poor-quality samples is unlikely to result in a large incremental yield. With a case definition of one positive smear, we recommend that bleach sedimentation is implemented in settings where work has been done to improve sputum collection practices. Sample quality can be confirmed with SCA. A pilot study should 
be undertaken prior to routine implementation of bleach sedimentation to determine whether the technique will be of sufficient clinical benefit in a particular setting.

\section{Acknowledgements}

We thank the Mindouli TB nursing and laboratory teams for undertaking and completing this study. We also thank the MSF country management team for negotiating the Memoranda of Understanding with the Ministry of Health authorities. Thank you to Kodzo Tukpo, Sylvie Cot, and Cecile de la Teysonniere, the laboratory supervisors. We also thank Sarah Venis and Stephanie Bartlett for editing assistance.

\section{Author details}

'Manson Unit, Médecins Sans Frontières UK, 67-74 Saffron Hill, London EC1N 8QX, UK. ${ }^{2}$ Mindouli Hospital, Pool Region, Republic of Congo. ${ }^{3}$ Epicentre, 5355 rue Crozatier, 75012 Paris, France.

\section{Authors' contributions}

$\mathrm{PH}$ conceived and carried out the study and wrote the first draft of the paper. PN was responsible for study implementation and protocol revision and commented on the draft. JG and MB performed data analysis and commented on the draft. VS designed the study, wrote the protocol, and commented on the draft. All authors read and approved the final manuscript.

\section{Competing interests}

The authors declare that they have no competing interests.

Received: 5 May 2010 Accepted: 21 September 2010 Published: 21 September 2010

\section{References}

1. WHO: 2009 update tuberculosis facts.[http://www.who.int/tb/publications/ 2009/tbfactsheet_2009update_one_page.pdf], (accessed July 10, 2010).

2. Cattamanchi A, Dowdy DW, Davis JL, Worodria W, Yoo S, Joloba M, Hopewell PC, Huang L: Sensitivity of direct versus concentrated sputum smear microscopy in HIV-infected patients suspected of having pulmonary tuberculosis. BMC Infect Dis 2009, 9:53.

3. Matee M, Mtei L, Lounasvaara T, Wieland-Alter W, Waddell R, Lyimo J, Bakari M, Pallangyo K, von Reyn CF: Sputum microscopy for the diagnosis of HIV-associated pulmonary tuberculosis in Tanzania. BMC Public Health 2008, 8:68

4. Kanaujia GV, Lam PK, Perry S, Brusasca PN, Catanzaro A, Gennaro ML: Integration of microscopy and serodiagnostic tests to screen for active tuberculosis. Int J Tuberc Lung Dis 2005, 9:1120-1126.

5. Eamranond $\mathrm{P}$, Jaramillo E: Tuberculosis in children: reassessing the need for improved diagnosis in global control strategies. Int J Tuberc Lung Dis 2001, 5:594-603.

6. Elliott AM, Halwiindi B, Hayes RJ, Luo N, Tembo G, Machiels L, Bem C, Steenbergen G, Pobee JO, Nunn PP: The impact of human immunodeficiency virus on presentation and diagnosis of tuberculosis in a cohort study in Zambia. J Trop Med Hyg 1993, 96:1-11.

7. Sakundarno M, Nurjazuli N, Jati SP, Sariningdyah R, Purwadi S, Alisjahbana B, van der Werf MJ: Insufficient quality of sputum submitted for tuberculosis diagnosis and associated factors, in Klaten district, Indonesia. BMC Pulmonary Med 2009, 8:16.

8. Klarkowski DB: The use of sputum cytology on Ziehl-Neelsen stained smears to improve pulmonary TB diagnosis. 2006, 37th Union World Conference on Lung Health, Paris, Abstract PC-61857-03 [poster discussion].

9. Steingart KR, Ng V, Henry M, Hopewell PC, Ramsay A, Cunningham J, Urbanczik R, Perkins MD, Aziz MA, Pai M: Sputum processing methods to improve the sensitivity of smear microscopy for tuberculosis: a systematic review. Lancet Infect Dis 2006, 6:664-674.

10. Ramsay A, Squire SB, Siddiqi K, Cunningham J, Perkins MA: The bleach microscopy method and case detection for tuberculosis control. Int $J$ Tuberc Lung Dis 2006, 10:256-258.

11. Bonnet M, Ramsay A, Githui W, Gagnidze L, Varaine F, Guerin PJ: Bleach sedimentation: an opportunity to optimize smear microscopy for tuberculosis diagnosis in settings of high prevalence of HIV. Clin Infect Dis 2008, 46:1710-1716.

12. Pandey A, Asthana AK: Sedimentation method, a good alternative to centrifugation for concentration of acid fast bacilli in developing countries: A preliminary study from western Uttar Pradesh. Indian J Med Microbiol 2009, 27:83-84.

13. Rasheed MU, Dechu T: An overnight sedimentation method: improving the diagnosis of tuberculosis when electrical centrifuge is not available. Trop Doct 2008, 38:78-79.

14. Githui WA, Matu SW, Muthami LN, Juma E: Improved diagnosis of ZiehlNeelsen smear negative tuberculosis using sodium hypochlorite sedimentation method. East Afr Med J 2007, 84:455-459.

15. Cattamanchi A, Davis JL, Pai M, Huang L, Hopewell PC, Steingart KR: Does bleach processing increase the accuracy of sputum smear microscopy for diagnosing pulmonary tuberculosis? An updated systematic review and meta-analysis. J Clin Microbiol 2010, 48(7):2433-9.

16. UNAIDS/WHO Working Group on Global HIV/AIDS and STI Surveillance: Epidemiological fact sheet on HIV and AIDS. Core data on epidemiology and response: Congo 2008 update. Geneva: UNAIDS/World Health Organization 2014, 2008

17. WHO: Health Action in Crises: Republic of the Congo (August 2007). Geneva: World Health Organization 2007.

18. Johnson BR, Remeikis NA: Effective shelf-life of prepared sodium hypochlorite solution. J Endod 1993, 19:40-43.

19. WHO: Revision of the case definition for sputum smear positive pulmonary TB: background document. Geneva: World Health Organization 2007.

20. Angeby KA, Hoffner SE, Diwan VK: Should the 'bleach microscopy method' be recommended for improved case detection of tuberculosis? Literature review and key person analysis. Int I Tuberc Lung Dis 2004, 8:806-815

21. WHO: Proposed reduction of number of smears for the diagnosis of pulmonary TB: background document. Geneva: World Health Organization 2007.

22. Khan MS, Dar O, Tahseen S, Godfrey-Faussett P: Judging respiratory specimen acceptability for AFB microscopy: visual vs. microscopic screening. Trop Med Int Health 2009, 14:571-575.

23. WHO: Laboratory service in tuberculosis control. Microscopy part I. Report WHO/TB/98.258. Geneva: World Health Organization 1998.

\section{Pre-publication history}

The pre-publication history for this paper can be accessed here: http://www.biomedcentral.com/1471-2334/10/276/prepub

\section{doi:10.1186/1471-2334-10-276}

Cite this article as: Hepple et al.: Direct microscopy versus sputum cytology analysis and bleach sedimentation for diagnosis of tuberculosis: a prospective diagnostic study. BMC Infectious Diseases 2010 10:276.

\section{Submit your next manuscript to BioMed Central and take full advantage of:}

- Convenient online submission

- Thorough peer review

- No space constraints or color figure charges

- Immediate publication on acceptance

- Inclusion in PubMed, CAS, Scopus and Google Scholar

- Research which is freely available for redistribution 